POR CONSIDERARLO DE INTERÉS PARA TODOS LOS ARCHIVISTAS Y ARCHIVEROS DE HABLA HISPANA, SE REPRODUCE A CONTINUACIÓN -DE MANERA ÍNTEGRAEL DISCURSO INTITULADO "ENTRE LA NOSTALGIA DE LA TRADICIÓN Y EL MITO DE LA MODERnidad", PRONUNCIADO POR LA DOCTORA ANTONia HEREDia HERRERA DURANTE LA CEREMONIA EN LA CUAL INGRESÓ -COMO ACADÉMICA correspondiente en Sevilla- en la Real Academia de Nobles Artes de Antequera, donde fue presentada por José Escalante Jiménez, ACADÉMICO DE NÚMERO. El ACTO, PRESIDIDO POR EL DIRECTOR DE ESA institución, Bartolomé Ruiz GonZÁlez, tuVO Lugar EL 20 DE JUNiO DE 2014 en el Real Monasterio de San Zoilo, en Antequera, España.

\title{
ENTRE LA NOSTALGIA DE LA TRADICIÓN Y EL MITO DE LA MODERNIDAD
}

Antonia Heredia Herrera

\section{Introducción}

Me siento doblemente honrada en esta ocasión, en primer lugar por la propia distinción y en segundo lugar por el hecho de ser propuesta por mi querido amigo Bartolomé Ruiz y mi compañero José Escalante. A los dos gracias de todo corazón y al resto de los académicos de la Real Academia de Nobles Artes de Antequera por aceptar la propuesta. Me siento distinguida como mujer y como archivera porque no siempre una y otra ocupan puestos de debido reconocimiento

Tengo sin embargo que confesar que he sido muy afortunada tanto en mi vida personal como en la profesional. Sin duda que en la primera hube de pagar muy temprano el tributo más elevado que hubiera supuesto pero tengo que admitir que fue compensado con un capital de afectos familiares y de amigos que me ha hecho millonaria. Por lo que respecta a mi vida profesional he tenido la suerte de ejercer como archivera en los distintos tipos de Archivos que componen la red de un sistema. Fueron 30 años en el Archivo General de Indias, luego casi 25 en el Archivo de la Diputación Provincial de Sevilla. Me faltaba el Archivo intermedio y fue 
Bartolomé Ruiz quien me comprometió para el Archivo General de Andalucía, donde permanecí durante ocho años hasta agotar el tiempo de mi carrera administrativa, completando así el circuito que afecta a la vida de los documentos.

Y para más abundamiento, mi trabajo ha sido reconocido y lo que es más gratificante, reconocido en vida.

\section{De archivística}

He dudado a la hora de preparar la intervención de hoy si inclinarme por la historia o por la archivística, una y otra de interés para mí, pero he optado por la segunda que ocupa el mayor tiempo de mi espacio profesional.

De archivística, de documentos, de Archivos, voy a tratar de hablaros con pretensión de hacerlos comprensibles y cercanos, porque no siempre existe diafanidad para los mismos y sus identidades quedan en más de una ocasión trastocadas y confundidas, más allá de lo pertinente. Mi discurso de ingreso va desprovisto de, al decir de la Pragmática de tratamientos y cortesías de Felipe II, de solemnidades inútiles y superfluas a favor de la cercanía y del conocimiento.

Todos sois conscientes que existen términos y conceptos que se imponen en determinados tiempos y se ponen de moda hasta el punto que su uso se generaliza. Uno de ellos es la contextualización -que ha venido a sustituir y ampliar la configuración- y por añadidura el contexto. Constatamos que el contexto, la contextualización son necesarios para conocer algo en profundidad, de tal manera que la contextualización se fundamenta en los atributos que hay que reconocer y en las relaciones que, establecidas, facilitan la configuración de ese algo. Y resulta evidente que dichas relaciones se insertan en los cambios sociales y tecnológicos que hoy afectan a todas las áreas de conocimiento.

La archivística no escapa a tal planteamiento, de aquí que trataré de contextualizarla para acercarla a quienes no son sus profesionales.

La archivística ha evolucionado de unas prácticas que se remontan en el tiempo y en el espacio, a Asiria, Egipto, hasta el establecimiento tardío de unos principios generales que han determinado una metodología específica aplicada a su doble objeto, los documentos de archivo y los Archivos como 
instituciones para el tratamiento, custodia y difusión de los primeros. Ha sido un largo camino, pasando por su dependencia de la diplomática, de la biblioteconomía, del derecho, de la historia, hasta alcanzar la autonomía. En cuanto a la última relación apuntada que ha sido muy fuerte, sin descartarla, ha cedido sitio a la relación con la administración, con la administración electrónica, hasta alcanzar hoy la categoría de ciencia con identidad propia dentro de las denominadas ciencias de la información o de la documentación. En el recorrido mencionado siempre se ha reconocido para ella una doble dimensión que busca el equilibrio entre la teoría y la práctica. La existencia de un vocabulario propio finalmente avala su condición científica.

La archivística y su doble objeto tienen unos atributos que la hacen reconocible, pero son las relaciones las que pueden ampliar su visibilidad para muchos. Esas relaciones son múltiples, entre otras con la información, con la memoria, con el patrimonio, con la historia, con la transparencia, con la administración electrónica, con los gobiernos abiertos, que no son las únicas relaciones, pero en esta ocasión me voy a quedar sólo con éstas.

Empezaré por los atributos de su objeto para continuar marcando las relaciones con las realidades de la secuencia mencionada para propiciar el acercamiento pretendido.

Durante muchos años hemos reconocido a la archivística como ciencia de los Archivos, y a renglón seguido hemos hablado de documentos, y por añadidura, de información. Hoy, conscientes de que no hay Archivos, -me refiero a las instituciones o centros-, sin documentos, coherentemente hemos seguido estimando el doble objeto, pero con un cambio de orden en su enumeración: documentos y Archivos, mejor que Archivos y documentos, sin descartar en tercer lugar nuestra atención a la información, acotada a la contenida en los referidos documentos.

En el sentido apuntado el cambio de orden tradicional queda patente en los títulos de las dos leyes andaluzas, la primera de 1984, de Archivos andaluces y la segunda, de 2011, de Documentos, Archivos y Patrimonio Documental.

El documento, objeto de la archivística, no es cualquier documento. En efecto, "documento", sin más, que no es sino un soporte sobre el que se registra una información, puede ser desde una lápida funeraria, una canción, una película, un periódico, etc., pero el documento con el apelativo de 
"archivo" es testimonio y prueba -ahora se dice evidencia- de acciones, de hechos de la sociedad, necesarios e inexcusables, como consecuencia de competencias y funciones atribuidas a instituciones, familias o personas. De aquí que los documentos testimonio de acciones creativas no son necesariamente documentos de archivo, salvo que formen parte de aquéllos. Ese documento de archivo, al que me refiero, se reconoce tanto en el espacio analógico como en el digital porque en uno y otro caso serán atributos o características indispensables para el mismo: la autenticidad, la integridad, la fiabilidad, la disponibilidad, características de las que otros documentos y la propia información carecen. Es tanto el administrativo con vigencia administrativa como el histórico -que no lo es por ser antiguo sino para el que se ha decidido y aplicado la conservación permanente, o como ahora se dice la conservación "a largo plazo". Y todo esto no es obstáculo para reconocer junto a los documentos de archivo otros testimonios que son también información, son también memoria, pero son distintos, sin perjuicio de que los archiveros, si estos últimos están en un Archivo tengan sobre ellos responsabilidad de custodia, tratamiento y difusión. Sería el caso de la colección de carteles de toros en el Archivo, del Ayuntamiento de San Sebastián de los Reyes o de la colección de postales en el Archivo del Ayuntamiento de Barcelona.

Del doble objeto de la archivística, el Archivo como institución es el lugar de custodia y tratamiento habitual y adecuado para esos documentos de archivo. Pero no siempre se acierta cuando llamamos Archivo a algo que no lo es. No hace mucho en la revista peruana Alerta archivística se daba la noticia sobre el primer Archivo histórico de modas de Yves Saint Laurent, y sin descartar la iniciativa lo que hay que reconocer es que la denominación quizá le queda holgada. ¿No sería mejor hablar de Museo? Lo que resulta del todo constatado es que el Archivo actualmente está sufriendo una notable transformación en su aspecto físico, estamos pasando de los edificios emblemáticos como la Lonja de mercaderes o el edificio de los Juzgados en Sevilla, o el convento de Santo Domingo en Jaén a unos potentes servidores sin que la responsabilidad de uso y de servicio debiera dejar de pertenecer al archivero y no a otro profesional.

En cuanto al objetivo de la archivística, permanece el servicio de los documentos y de la información en ellos contenida. Servicio que, restringido 
durante años a los historiadores, ha cobrado amplitud a partir, en primer lugar, de una ampliación a la administración y a cualquier ciudadano, y en segundo lugar, a partir de una apertura que lleva a la facilitación sin esperar la demanda. Este dimensionamiento del servicio para el que la regulación del acceso es determinante tiene su expresión más contundente en los múltiples proyectos de digitalización, siendo significativos el Portal de Archivos Españoles (Pares) del Ministerio de Cultura, o el proyecto de los libros sacramentales en el país vasco. Cualquiera a cualquier hora, desde cualquier lugar, puede entrar en el Archivo sin esperar a que su puerta se abra, y consultar los documentos de su interés, sentado en el sillón de su casa.

Y de los atributos paso a las relaciones enumeradas. Información, memoria y patrimonio quizá constituyan el primer conjunto de esas relaciones con los archivos.

\section{Los archivos y la información}

En efecto, hay dos relaciones potentes del documento de archivo con la información y con la memoria, relaciones que en ningún momento pueden llevarnos a la identificación porque la sinonimia no tiene cabida en este caso.

De hecho, la información no tiene por qué estar ligada al documento, tiene vida propia, puede cambiar, ampliarse, actualizarse, tergiversarse. El documento de archivo, sin embargo, está ligado a una información concreta que no puede variar porque la acción que testimonia y prueba no varía, y de hecho si esa información la cambiamos daríamos lugar a la trasgresión, al delito. El documento de archivo que tiene voluntad de verdad es reproducción fija de una información concreta que puede no ser verdadera si su autor ha tenido voluntad de falsearla.

\section{Los archivos y la memoria}

En cuanto a la memoria, desde antiguo, como apelativo, se ha aplicado a los Archivos y a los documentos de archivo. Hemos llamado "memoria de la sociedad" al conjunto de los Archivos, "memoria de la cristiandad" al 
Archivo vaticano, "memoria de un continente" al Archivo de Indias, "memoria de Antequera" al Archivo municipal de Antequera. Apelativo de "memoria", que dimensionado hoy, ha llevado a algunos, en el entorno de la sinonimia, a ciertos equívocos. Porque ocurre que mientras la memoria olvida, selecciona y distorsiona, el documento de archivo la fija, la acota, la hace estable.

Empecemos por definir la memoria, de la que podemos decir que: "es la retención del recuerdo de algo pasado, reciente o antiguo" de tal manera que su percepción no es evidencia sino aproximación a una realidad. Siempre es retrospectiva y alejada de la exclusiva vinculación a un determinado tiempo y espacio.

Actualmente el concepto de "memoria" ha cobrado, por una parte, dimensión política y social y, por otra, dimensionamiento conceptual abanderado por el posmodernismo.

En el entorno político, la expresión "memoria histórica" se utiliza-desde mi punto de vista- en más de un país, con falta de propiedad, porque la reduce en el espacio y la acorta en el tiempo, al limitarla a un período próximo y reciente. En el entorno social no podemos dejar de reconocer la violación de derechos humanos en determinados momentos y el derecho de las víctimas a conocer la verdad para reclamar justicia, de aquí la necesidad de liberar el acceso a los archivos con documentos que testimonian la represión.

Por su parte la corriente posmodernista que ha trascendido a la archivística, a través de la obra del francés Jacques Derrida y de sus seguidores, han sido quienes dan preferencia al término y concepto de "memoria" por encima del de "archivo": la obsesión para ellos es la memoria "sin rastros de olvido". De aquí que denuncien la ausencia de mujeres, de gays, de gitanos, en esa memoria que identifican con los Archivos. Y ocurre que tal identificación parece hacer culpables a los archiveros por haber dedicado una atención monográfica a los documentos de la administración, de las instituciones relevantes y de las familias y personas con poder, dejando a un lado la recuperación de documentos pertenecientes a grupos sociales marginales. Nuestra posición, que no nuestra defensa, porque los archiveros no somos culpables, es que nuestra atención prioritaria son sin duda los documentos de archivo, y quizá esos grupos no los han producido directamente y por lo tanto, no han existido para guardarlos, tratarlos y conservarlos, sin perjuicio de que existan otros testimonios, otros documentos que atestigüen su 
existencia y sus comportamientos. No me cabe la menor duda que cuando los documentos de archivo existen y se conservan, parte, que no toda la memoria de esos grupos marginales, está a salvo. Las leyes de Indias, muchas series documentales conservadas en el Archivo General de Indias o en los documentos de los Archivos relacionados con instituciones afines a los derechos humanos, como es el caso, en España, de los defensores del pueblo, de los institutos de la mujer o de los tribunales superiores de justicia militar, entre otros, conservan documentos de archivo que son parte de la memoria de esos grupos marginales y, como tal, también parte de la memoria social.

Cuando hoy se habla de centros de la memoria, valgan por caso el denominado de la "Memoria histórica" o el centro de la memoria histórica de la Fundación Nelson Mandela, no pueden identificarse con los Archivos. A diferencia de éstos, se crean, se inventan en un momento oportuno; no nacen, ni se forman de manera natural como suele ocurrir con los Archivos; se fuerza, se determina su creación. En estos centros de la memoria hay menos documentos de archivo que documentos como suelen ser los de tradición oral, los periódicos, las fotografías, las letras de canciones, los carteles, incluso objetos de cualquier naturaleza. Porque existen países o tiempos; en el caso de regímenes represivos como el del apartheid sudafricano, el gobierno de Pinochet en Chile o la guerra civil en España, en los que la inexistencia, la pérdida o la destrucción de documentos de archivo han determinado el uso inevitable de otros testimonios, y en estos casos los centros de la memoria sustituyen a los Archivos sin tener que identificarse con ellos. Posicionamiento que muchos de mis colegas no comparten cuando los denominan Archivos.

De aquí que la plena identificación de la memoria con los documentos de archivo y con las instituciones de archivo puede distorsionar conceptos y situaciones. Los documentos son memoria, pero mientras las memorias son múltiples dependiendo de quién las elabore, los documentos de archivo son unos, sin posibilidad de variación ni de alteración. Otra cosa serán los usos que hagamos de los documentos para la elaboración de las distintas memorias. La memoria social por otra parte no puede construirse sólo con documentos de archivo, del mismo modo que los documentos de archivo son sólo parte de la memoria, y los Archivos siendo custodios de la memoria 
no lo son en exclusividad. Me llena de desasosiego cuando oigo decir que se va a crear un Archivo de la memoria, ¿qué Archivo no lo es?

En el contexto archivístico para la constitución de la memoria, a sabiendas de que la misma no puede ser sino selectiva porque no se puede recordar todo, siempre ha de recurrirse a un proceso de valoración para seleccionar lo más importante. Ahora bien, ¿qué es lo más importante? ¿cuáles son los documentos esenciales? De aquí que la memoria que trasmiten los documentos de archivo está relacionada con una función archivística importante y comprometida que tras valorarlos nos lleva a seleccionar marcando relaciones con la memoria y con el patrimonio documental.

\section{Los archivos y el patrimonio}

Y sin duda que los documentos de archivo tienen que ver con el patrimonio documental.

El "patrimonio" es un término al que trasciende el concepto de "legado", de conjunto de bienes de interés que ha de conservarse de forma permanente para trasmitir a generaciones venideras. Patrimonios hay muchos y diversos. El arqueológico, el bibliográfico, el documental, el cultural, el fotográfico, el marino, el medioambiental, el digital, etc., pero sólo el documental es el directamente relacionado con la archivística.

Dicho patrimonio, acotado en la Ley de Patrimonio Histórico del 85, está constituido por todos los documentos de archivo de entidades públicas y por los privados a partir de unos determinados plazos. Para los primeros el tiempo no cuenta; según dicha ley, cualquier documento de una institución pública desde el momento de producirse forma parte del referido patrimonio, y sin duda que casa mal tal amplitud con el concepto de patrimonio que acabamos de señalar, cuando la eliminación resulta obligada para un porcentaje muy elevado de dichos documentos.

En cuanto a los documentos privados integrantes del patrimonio documental, también existe cierta distorsión, en cuanto los plazos establecidos para tal estimación arrancan de los 50 o 100 años cumplidos, plazos que por amplios pueden dar lugar a que los citados documentos no alcancen el término temporal establecido para la estimación de históricos. Esa atención tardía al patrimonio documental privado puede en cierta 
medida dar razón al movimiento posmodernista defensor de la memoria total sin rastros de olvido.

Estos planteamientos, establecidos por una ley que ha quedado desfasada al no ser modificada, continúan obligando a toda la legislación archivística española posterior, que si antes del 78 era nula, desde entonces es muy numerosa. Y está ocurriendo que la referida acotación documental de patrimonio está llevando a reconocimientos legales que deberían plantear la repulsa cuando se llega a decir "la eliminación legal de documentos constitutivos del patrimonio documental".

\section{Los archivos y la transparencia}

Y continuemos con otra de las relaciones: la de los archivos con la transparencia.

La información es poder, decíamos ayer; hoy, avanzando, reconocemos que la información facilita el ejercicio de la democracia a través de lo que hoy conocemos como transparencia.

"Transparencia" es un término y concepto importado del contexto anglosajón que en algunos países ha nacido ligado a la corrupción, pero también se afirma que se exige y consolida a partir de la pérdida de legitimidad de la clase política. Lo que resulta evidente es que la transparencia se hace patente a la hora de las administraciones electrónicas dada la posibilidad que brindan las tecnologías para la difusión y sobre todo para que los ciudadanos interactúen con la administración. La transparencia está ligada a los cambios sociales que exigen derecho a la información y, por tanto, a la regulación del acceso. De aquí que los documentos de archivo ligados a una información fiable sean elementos indispensables a la hora de regular la transparencia, para lo que habrá que distinguir perfectamente entre documentos de archivo e información, porque una cosa y otra -como ya vimos- no son lo mismo y ciertamente esto no siempre queda nítido en los textos legales que se están elaborando. No es lo mismo facilitar un documento que simplemente hay que localizar que elaborar una información sobre algo.

Se insiste una y otra vez en que los Archivos y sus documentos son substanciales en este contexto. Pero no perdamos de vista que la transparencia y el acceso pueden tener efectos disuasorios. De aquí que 
este papel, para ser bien desempeñado, exija por parte de las organizaciones públicas la producción de documentos con los atributos que les hemos reconocido: autenticidad, fiabilidad, integridad y disponibilidad. Porque si no se producen -es decir, no se testimonian acciones por comprometidas-, o se producen y no se transfieren a los Archivos, o se producen y no son accesibles para los ciudadanos, la transparencia quedará en caricatura. En esta línea, la Federación Española de Asociaciones de Archiveros, Bibliotecarios, Arqueólogos, Museólogos y Documentalistas (ANABAD), hace dos meses escasos denunciaba la falta de un reglamento para la aplicación de la ley de transparencia porque el Ministerio de Asuntos Exteriores sigue denegando documentos con más de 30 años.

\section{Los archivos y la historia}

Nos referimos al principio a la dependencia temprana y persistente de la archivística con la historia. No podemos dejar de reconocer que la historia es reconstrucción antes que restauración, reparación o invención, y esa reconstrucción será difícil sin documentos de archivo. El pasado que es ayer y antes de ayer no podrá perder mañana su condición de oscuro porque, sin documentos, la historia no le devolverá la claridad que le falta. Esa relación estrecha ha determinado que, durante tiempo, hablemos casi con exclusividad de Archivos históricos, de formación historicista para los archiveros y de limitación de usuarios a los historiadores. Esta vinculación ha determinado hasta ahora la atribución gestora sobre ellos a entidades de cultura (ministerios, consejerías), sin embargo, el giro de lo histórico a lo administrativo -como vamos a ver- está reclamando vinculaciones gestoras más adecuadas en tanto en cuanto -como tendremos ocasión de comprobar- el modelo de gestión documental, que siempre es transversal, lo eligen las organizaciones.

Por otra parte, no cabe duda que los documentos de archivo siempre han tenido una vinculación inexcusable con la acción de la administración, en tanto en cuanto siempre han sido su testimonio y prueba, pero su protagonismo no ha existido durante muchos años hasta pasado el tiempo suficiente para estimarlos históricos. Sin embargo, insisto, es el concepto de 
administrativo antes que el de histórico el que imprime carácter al documento de archivo. De hecho todos los documentos de archivo son administrativos, en el sentido más amplio del término, pero no todos alcanzan la categoría de históricos porque los documentos de archivo - vuelvo a repetirlo- no son tales porque estén en un Archivo o sean antiguos, sino que han nacido como algo obligado, necesario y son testimonio y prueba de transacciones necesarias y establecidas por la sociedad. Ahora bien, no existe para todos un limite cronológico único entre lo administrativo y lo histórico. Los plazos varían y tienen una relación directa con la vigencia administrativa que como bien sabéis no es única.

Hasta la pasada década de los sesenta, lo histórico se imponía. La atención profesional sólo era para los Archivos históricos de la administración del Estado. Serán los archiveros, sobre todo los de corporaciones locales, los que en la década siguiente empiecen a dar justo valor a los Archivos administrativos y de aquí el cambio de signo de la relación que nos ha llevado decididamente de la historia a la administración.

\section{Los archivos y la e-administración y los gobiernos abiertos}

Y junto a esto la sintonía con los cambios sociales y la incorporación de las tecnologías de la información, del conocimiento y de la convergencia universal, quizá aquéllos favorecidos por éstas. Cambios sociales que dan un protagonismo cada vez mayor a los ciudadanos, de aquí la frecuente alusión a la corresponsabilidad, aunque también pueden acarrear abusos. El marco para esta sintonía que está favorecido por la globalización, trasciende a la interoperabilidad, se manifiesta en el acceso y la transparencia, se evidencia en una legislación que se amplía y se desarrolla día a día. El punto de partida es la ley 11/2007 de 22 de junio de acceso electrónico de los ciudadanos a las actuaciones de los servicios públicos, la cual no es sino la voluntad de implantar una administración electrónica que facilite la comunicación entre administradores y ciudadanos.

En este contexto el concepto de apertura cobra sentido, dando un paso hacia delante con el denominado gobierno abierto por el que de la comunicación con los ciudadanos se llega a la participación y 
colaboración con dichos ciudadanos en la administración pública a favor del fortalecimiento democrático. En este sentido la teoría va por delante de la práctica y nos queda mucho camino que recorrer.

La abundante normativa sobre la e-administración habla decididamente de gestión documental, de documentos de archivo y de Archivos o repositorios electrónicos, de modelo de gestión documental. Gestión documental que hoy no empieza a partir del ingreso de los documentos en los Archivos sino que se integra en las políticas de gobiernos electrónicos y alude al nuevo paradigma tecnológico asociado a la prestación de servicios por parte de los poderes públicos para entre otros objetivos proveer de más y mejores servicios a los ciudadanos, proveer de puntos de acceso unificados y sencillos para satisfacer múltiples necesidades informativas, para aumentar la participación ciudadana, reducir costos y aumentar la transparencia. ¡No es poca cosa! Y ocurre que los procesos de la gestión documental electrónica no son otros que las funciones que hasta ahora los archiveros tenían como propias y ahora van a ser compartidas, porque existe voluntad de producir, mantener y utilizar documentos auténticos, fiables, íntegros y disponibles. Hasta el punto que la archivística ha dejado de ser una disciplina sólo para los archiveros.

\section{Reflexión final}

Quizá lo expuesto haya supuesto alguna precisión para acercarse a conocer el entorno donde hoy trabajamos los archiveros, pero no quiero terminar sin algún comentario final.

Hoy cualquier disciplina defiende en su presentación el marchamo de "nueva". Lo tradicional suena a caduco, a obsoleto, hasta el extremo manifestado por alguna corriente de pensamiento, que trata de desnaturalizar aquello que la sociedad entiende como natural marcando una desconfianza total hacia los conocimientos objetivos, hacia las verdades universales, llevando a sus seguidores a estimar nuevos todos sus planteamientos partiendo de un olvido generalizado, convertido en inopia, para justificar la novedad que pretenden.

En nuestro caso, las expresiones "archivística nueva", "archivística posmodernista", "archivística digital", parecen haber roto moldes. Sin 
embargo, desde mi punto de vista, la novedad y modernidad aplicadas a la archivística habrá que matizarlas. Sería un error pensar que partimos de cero.

Entiendo que la archivística de hoy en su base, en su esencia, no puede ser diferente, distinta -incluso cuando hablemos de documentos electrónicossi sus principios permanecen, si se mantiene su objeto y si su objetivo no es otro que el servicio y disfrute de los documentos para la sociedad, no sólo para los historiadores. Lo realmente cambiante es su metodología, que al amparo de las nuevas tecnologías de la información y del conocimiento van a afectar a todas las funciones archivísticas, muchas de las cuales serán abiertas, corresponsables y compartidas con otros profesionales y usuarios. Lo que no cabe cuestionarse es que la archivística ha evolucionado naturalmente y se ha desarrollado sensible y aceleradamente con ayuda de esas tecnologías y al hilo de las exigencias sociales.

Insisto: el recorrido entre la tradición y la novedad, como manifestación de la modernidad, siendo largo ha sufrido una aceleración en los últimos años que casi no nos da ocasión a reflexionar y no faltan quienes -como he dicho- situándose en la última etapa del camino, no quieren saber nada de la tradición. Sin embargo, lo nuevo de hoy parte de muchas de las aportaciones de ayer, e ignorarlas y no saber construir sobre ellas es una temeridad que puede ser irremediable y llevarnos a la pérdida de nuestra identidad.

La novedad exige una continua actualización a partir de renovadas preguntas que nos permitan abordar los cambios de hoy y los por venir.

Cambios que afectan al vocabulario y con su uso al lenguaje que se enriquece y evoluciona y ha de convivir con vocabularios de otros profesionales corresponsables; cambios que afectan a la normalización que va sustituyendo poco a poco la discrecionalidad de muchos de nuestros métodos; cambios que afectan al servicio y a sus usuarios que se han hecho más generales y más universales; cambios que han sustituido el aislamiento de los Archivos y de los archiveros por la integración en la organización y en su gestión; cambios que han hecho evolucionar el perfil del archiverohistoriador y del archivero-conservador al archivero-gestor y al archiverocomunicador.

Entiendo que es fácil reconocer -al menos para mí- que hemos pasado de la tradición a la modernidad sin sobresaltos, aunque con cambios acelerados. 
Quizá esté ocurriendo algo parecido en otras áreas de conocimiento.

Espero que la archivística, los documentos de archivo y los Archivos a partir de hoy sean más familiares a quienes alguna o poca relación han tenido con ellos.

Para concluir, reitero mi profundo agradecimiento a sus señorías por haberme hecho el honor de considerarme entre sus miembros correspondientes.

Muchas gracias.

\section{Bibliografía:}

* Pragmática en que se da la orden y forma que se ha de tener y guardar en los tratamientos y cortesías de palabra y por escrito..., San Lorenzo 8 octubre 1586 (impreso) AGI.

Cruz Mundet, J.R., La gestión documental en las Organizaciones, Ediciones Pirámide, 2006

Guichot, E., Transparencia y acceso a la información en España: análisis y propuestas legislativas, Madrid, Fundación alternativas, 2011

Heredia Herrera, A., "Historia, memoria y gestión en el contexto archivístico", en La utilidad de los archivos, estudios en bomenaje a Manuel Vaquerizo Gil, PubliCan, ediciones de la Universidad de Cantabria, 2011

2012, núm. 1, PP.15-28

, "La Archivística a debate", Boletín de ANABAD, LXII, , Lenguaje y vocabulario archivistico: algo más que un diccionario, Junta de Andalucía, Consejería de Cultura, 2011

, Manual de Archivistica básica: gestión y sistemas, Universidad autónoma de Puebla (México), 2013

López. Gómez, P.; Gallego Domínguez, El documento de archivo. Un estudio, Universidade da Coruña, 2007

Mendoza Navarro, A., Documentos electrónicos de archivo. Una visión integradora, Lima, Biblioteca Nacional, 2009 